\title{
Jerk, snap and the cosmological equation of state
}

\author{
Matt Visser \\ School of Mathematical and Computing Sciences, Victoria University of Wellington, \\ PO Box 600, Wellington, New Zealand \\ E-mail: matt.visser@mcs.vuw.ac.nz
}

Received 27 February 2004

Published DD MMM 2004

Online at stacks.iop.org/CQG/21/1 (DOI: 10.1088/0264-9381/21/0/000)

\section{Abstract}

Taylor expanding the cosmological equation of state around the current epoch

$$
p=p_{0}+\kappa_{0}\left(\rho-\rho_{0}\right)+\left.\frac{1}{2} \frac{\mathrm{d}^{2} p}{\mathrm{~d} \rho^{2}}\right|_{0}\left(\rho-\rho_{0}\right)^{2}+O\left[\left(\rho-\rho_{0}\right)^{3}\right]
$$

is the simplest model one can consider that does not make any a priori restrictions on the nature of the cosmological fluid. Most popular cosmological models attempt to be 'predictive', in the sense that once some a priori equation of state is chosen the Friedmann equations are used to determine the evolution of the FRW scale factor $a(t)$. In contrast, a 'retrodictive' approach might usefully take observational data concerning the scale factor, and use the Friedmann equations to infer an observed cosmological equation of state. In particular, the value and derivatives of the scale factor determined at the current epoch place constraints on the value and derivatives of the cosmological equation of state at the current epoch. Determining the first three Taylor coefficients of the equation of state at the current epoch requires a measurement of the deceleration, jerk and snap-the second, third and fourth derivatives of the scale factor with respect to time. Higher-order Taylor coefficients in the equation of state are related to higher-order time derivatives of the scale factor. Since the jerk and snap are rather difficult to measure, being related to the third and fourth terms in the Taylor series expansion of the Hubble law, it becomes clear why direct observational constraints on the cosmological equation of state are so relatively weak; and are likely to remain weak for the foreseeable future.

PACS number:

\section{Introduction}

This paper develops a 'phenomenological' approach to the equation of state (EOS) of the cosmological fluid, and investigates what would have to be done in order to observationally 
determine the EOS. Even at the linearized level, where

$$
p=p_{0}+\kappa_{0}\left(\rho-\rho_{0}\right)+O\left[\left(\rho-\rho_{0}\right)^{2}\right],
$$

the first nontrivial coefficient in the EOS will be seen to be related to the cosmological jerkthe third derivative of the scale factor with respect to time, and thence to the third-order term in the Taylor series expansion of the Hubble law. This is the fundamental reason why observational determinations of the EOS are relatively poor, and why it is possible to choose so many wildly differing a priori models for the EOS that nevertheless give good agreement with the coarse features of the present epoch.

More generally, if we describe the cosmological equation of state in terms of a Taylor series expansion around the current epoch

$$
p=p_{0}+\left.\sum_{n=1}^{N-1} \frac{1}{n !} \frac{\mathrm{d}^{n} p}{\mathrm{~d} \rho^{n}}\right|_{0}\left(\rho-\rho_{0}\right)^{n}+O\left[\left(\rho-\rho_{0}\right)^{N}\right],
$$

then the $n$ th-order Taylor coefficient

$$
\left.\frac{\mathrm{d}^{n} p}{\mathrm{~d} \rho^{n}}\right|_{0}
$$

will be shown to depend on the $(n+2)$ th time derivative of the scale factor

$$
\left.\frac{\mathrm{d}^{n+2} a(t)}{\mathrm{d} t^{n+2}}\right|_{0},
$$

and thence to the $(n+2)$ th term, the $O\left(z^{n+2}\right)$ term, in the Taylor expansion of the Hubble law.

In most attempts at cosmological model building one takes a FRW cosmology

$$
\mathrm{d} s^{2}=-c^{2} \mathrm{~d} t^{2}+a(t)^{2}\left[\frac{\mathrm{d} r^{2}}{1-k r^{2}}+r^{2}\left(\mathrm{~d} \theta^{2}+\sin ^{2} \theta \mathrm{d} \phi^{2}\right)\right],
$$

plus the conservation of stress-energy

$$
\dot{\rho} a^{3}+3[\rho+p] a^{2} \dot{a}=0,
$$

and chooses some a priori equation of state

$$
\rho=\rho(p), \quad \text { or } \quad p=p(\rho),
$$

to derive $\rho(a)$, and equivalently $p(a)$. The Einstein equations then reduce to the single Friedmann equation, which can be written in the form

$$
\dot{a}=\sqrt{\frac{8 \pi G_{N} \rho(a) a^{2}}{3}-k},
$$

and used to determine $a(t)$. (See, for example, any standard text such as [1-3].)

In contrast, let us assume we have a FRW universe with good observational data on $a(t)$ - in Weinberg's terminology we have a good 'cosmography' [1]. In this situation we can use the Einstein equations in reverse to calculate the energy density $\rho(t)$ and pressure $p(t)$ via

$$
\begin{aligned}
& 8 \pi G_{N} \rho(t)=3 c^{2}\left[\frac{\dot{a}^{2}}{a^{2}}+\frac{k c^{2}}{a^{2}}\right], \\
& 8 \pi G_{N} p(t)=-c^{2}\left[\frac{\dot{a}^{2}}{a^{2}}+\frac{k c^{2}}{a^{2}}+2 \frac{\ddot{a}}{a}\right] .
\end{aligned}
$$

Under mild conditions on the existence and nonzero value of appropriate derivatives, we can appeal to the inverse function theorem to assert the existence of a $t(\rho)$ or $t(p)$ and hence, in principle, deduce an observational equation of state

$$
\rho(p)=\rho(t=t(p)), \quad p(\rho)=p(t=t(\rho)) .
$$


In view of the many controversies currently surrounding the cosmological equation of state, and the large number of speculative models presently being considered, such an observationally driven reconstruction is of interest in its own right.

Now in observational cosmology we do not have direct access to $a(t)$ over the entire history of the universe-we do, however, have access (however imprecise) to the current value of the scale factor and its derivatives, as encoded in the Hubble parameter, deceleration parameter, etc. This more limited information can still be used to extract useful information about the cosmological equation of state, in particular it yields information about the present value of the $w$-parameter and the slope parameter $\kappa_{0}$ defined as

$$
w_{0}=\left.\frac{p}{\rho}\right|_{0}, \quad \kappa_{0}=\left.\frac{\mathrm{d} p}{\mathrm{~d} \rho}\right|_{0} .
$$

The value of the $w$-parameter in particular has recently become the centre of considerable interest, driven by speculation that $w_{0}<-1$ is compatible with present observations. Such a value of $w_{0}$ would correspond to present-day classical and cosmologically significant violations of the null energy condition. The associated 'phantom matter' (almost identical to the notion of 'exotic matter' in the sense of Morris and Thorne [4]) leads to a cosmological energy density that is future increasing rather than future decreasing. (See, for example, $[5,6]$ ). If $w(t)$ subsequently remains strictly less than -1 , this will lead to a 'big rip' [7]—-the catastrophic infinite expansion of the universe in finite elapsed time.

Unfortunately, it is very difficult to measure $w_{0}$ and $\kappa_{0}$ with any accuracy-I will make this point explicit by relating the measurement of $w_{0}$ to the deceleration parameter, and the measurement of $\kappa_{0}$ to the 'jerk' of the cosmological scale factor-the third derivative with respect to time.

For related comments see [8-13]. The 'cubic' term of Chiba and Nakamura [8] is identical to the jerk, as is the 'statefinder' variable called $r$ by Sahni et al [9-11]. The other 'statefinder' variable (called $s$, not to be confused with the snap) is a particular linear combination of the jerk and deceleration parameters. Padmanabhan and Choudhury [12] have also emphasized the need for constructing models for the cosmological fluid that are unprejudiced by a priori theoretical assumptions. A good recent survey of the status of the cosmological fluid is [13].

\section{Hubble, deceleration, jerk and snap parameters}

It is standard terminology in mechanics that the first four time derivatives of position are referred to as velocity, acceleration, jerk and snap ${ }^{1}$. In a cosmological setting this makes it appropriate to define Hubble, deceleration, jerk and snap parameters as

$$
\begin{aligned}
& H(t)=+\frac{1}{a} \frac{\mathrm{d} a}{\mathrm{~d} t} \\
& q(t)=-\frac{1}{a} \frac{\mathrm{d}^{2} a}{\mathrm{~d} t^{2}}\left[\frac{1}{a} \frac{\mathrm{d} a}{\mathrm{~d} t}\right]^{-2}, \\
& j(t)=+\frac{1}{a} \frac{\mathrm{d}^{3} a}{\mathrm{~d} t^{3}}\left[\frac{1}{a} \frac{\mathrm{d} a}{\mathrm{~d} t}\right]^{-3}, \\
& s(t)=+\frac{1}{a} \frac{\mathrm{d}^{4} a}{\mathrm{~d} t^{4}}\left[\frac{1}{a} \frac{\mathrm{d} a}{\mathrm{~d} t}\right]^{-4} .
\end{aligned}
$$

1 Jerk (the third time derivative) is also sometimes referred to as jolt. Less common alternative terminologies are pulse, impulse, bounce, surge, shock and super-acceleration. Snap (the fourth time derivative) is also sometimes called jounce. The fifth and sixth time derivatives are sometimes somewhat facetiously referred to as crackle and pop. 
The deceleration, jerk and snap parameters are dimensionless, and we can write

$$
\begin{gathered}
a(t)=a_{0}\left\{1+H_{0}\left(t-t_{0}\right)-\frac{1}{2} q_{0} H_{0}^{2}\left(t-t_{0}\right)^{2}+\frac{1}{3 !} j_{0} H_{0}^{3}\left(t-t_{0}\right)^{3}\right. \\
\left.+\frac{1}{4 !} s_{0} H_{0}^{4}\left(t-t_{0}\right)^{4}+O\left(\left[t-t_{0}\right]^{5}\right)\right\} .
\end{gathered}
$$

In particular, at arbitrary time $t$

$$
w(t)=\frac{p}{\rho}=-\frac{H^{2}(1-2 q)+k c^{2} / a^{2}}{3\left(H^{2}+k c^{2} / a^{2}\right)}=-\frac{(1-2 q)+k c^{2} /\left(H^{2} a^{2}\right)}{3\left[1+k c^{2} /\left(H^{2} a^{2}\right)\right]} .
$$

While observation is currently not good enough to distinguish between the three cases $k=-1 / 0 /+1$ with any degree of certainty, there is nevertheless widespread agreement that at the present epoch $H_{0} a_{0} / c \gg 1$ (equivalent to $\left|\Omega_{0}-1\right| \ll 1$ ).

Warning. From a theoretical perspective, $H_{0} a_{0} / c \gg 1$ is a generic prediction of inflationary cosmology - this is not the same as saying that cosmological inflation predicts $k=0$. What generic cosmological inflation predicts is the weaker statement that for all practical purposes the present day universe is indistinguishable from a $k=0$ spatially flat universe. If our universe happens to be a topologically trivial $k=0$ FRW cosmology, then we will never be able to prove it. Simply as a matter of formal logic, all we will ever be able to do is to place increasingly stringent lower bounds on $H_{0} a_{0}$, but this will never rigorously permit us to conclude that $k=0$. The fundamental reason for this often overlooked but trivial observation is that a topologically trivial $k=0$ FRW universe can be mimicked to arbitrary accuracy by a $k= \pm 1$ FRW universe provided the scale factor is big enough ${ }^{2}$. In contrast, if the true state of affairs is $k= \pm 1$, then with good enough data on $H_{0} a_{0}$ we will in principle be able to determine upper bounds which (at some appropriate level of statistical uncertainty) demonstrate that $k \neq 0$. Also note that even in inflationary cosmologies it is not true that $H(t) a(t) / c \gg 1$ at all times, and in particular this inequality may be violated (and often is violated) in the pre-inflationary epoch.

Now the $w$-parameter in cosmology is related to the Morris-Thorne exoticity parameter [4] which was introduced by them to characterize the presence of 'exotic matter', matter violating the null energy condition (NEC):

$$
\xi=\frac{\rho+p}{|\rho|}=\operatorname{sign}(\rho)[1+w]=\frac{2}{3} \operatorname{sign}(\rho) \frac{1+q+k c^{2} /\left(H^{2} a^{2}\right)}{1+k c^{2} /\left(H^{2} a^{2}\right)} .
$$

Thus if $w<-1$ and $\rho>0$ we have $\xi<0$ and the NEC is violated. In contrast, if $w<-1$ but $\rho<0$ we have $\xi>0$, the NEC is satisfied but the weak energy condition (WEC) is violated. That is, 'phantom matter' (matter with $w<1$ ) is not quite the same as 'exotic matter' (for which $\xi<0$ ), but the two are intimately related.

Accepting the approximation that $H_{0} a_{0} / c \gg 1$ we have

$\rho_{0} \approx \frac{3}{8 \pi G_{N}} H_{0}^{2}>0, \quad w_{0} \approx-\frac{\left(1-2 q_{0}\right)}{3}, \quad$ and $\quad \xi_{0} \approx \frac{2}{3}\left(1+q_{0}\right)$,

so that in this situation the $w_{0}$-parameter and exoticity parameter $\xi_{0}$ are intimately related to the deceleration parameter $q_{0}$. In particular if $w_{0}<-1$, so that the universe is at the current epoch dominated by 'phantom matter', we also (because in this approximation $\rho_{0}$ is guaranteed to be positive) have $\xi_{0}<0$ so that at the current epoch this phantom matter is also

2 If the universe has nontrivial spatial topology there is a possibility of using the compactification scale, which might be (but does not have to be) much smaller than the scale factor, to indirectly distinguish between $k=-1 / 0 /+1$. 
'exotic matter'. Exotic matter is powerful stuff: apart from possibly destroying the universe in a future 'big rip' singularity [7], if the exotic matter clumps to any extent there is real risk of even more seriously bizarre behaviour - everything from violations of the positive mass condition (that is, objects with negative asymptotic mass), through traversable wormholes, to time warps $[4,14-17]$.

\section{Taylor series equation of state}

Linearize the cosmological EOS around the present epoch as

$$
p=p_{0}+\kappa_{0}\left(\rho-\rho_{0}\right)+O\left[\left(\rho-\rho_{0}\right)^{2}\right] .
$$

To calculate $\kappa_{0}$ we use

$$
\kappa_{0}=\frac{\mathrm{d} p /\left.\mathrm{d} t\right|_{0}}{\mathrm{~d} \rho /\left.\mathrm{d} t\right|_{0}}
$$

where numerator and denominator can be obtained by differentiating the Friedmann equations for $\rho(t)$ and $p(t)$. It is easy to see that at all times, simply from the definition of deceleration and jerk parameters, we have

$$
\begin{aligned}
& 8 \pi G_{N} \frac{\mathrm{d} \rho}{\mathrm{d} t}=-6 c^{2} H\left[(1+q) H^{2}+\frac{k c^{2}}{a^{2}}\right], \\
& 8 \pi G_{N} \frac{\mathrm{d} p}{\mathrm{~d} t}=2 c^{2} H\left[(1-j) H^{2}+\frac{k c^{2}}{a^{2}}\right],
\end{aligned}
$$

leading to

$$
\kappa_{0}=-\frac{1}{3}\left[\frac{1-j_{0}+k c^{2} /\left(H_{0}^{2} a_{0}^{2}\right)}{1+q_{0}+k c^{2} /\left(H_{0}^{2} a_{0}^{2}\right)}\right],
$$

which approximates (using $H_{0} a_{0} / c \gg 1$ ) to

$$
\kappa_{0}=-\frac{1}{3}\left[\frac{1-j_{0}}{1+q_{0}}\right] .
$$

The key observation here is that to obtain the linearized equation of state you need significantly more information than the deceleration parameter $q_{0}$; you also need to measure the jerk parameter $j_{0}$. If the only observations you have are measurements of the deceleration parameter then you can of course determine $w_{0}=p_{0} / \rho_{0}$, but this is not an equation of state for the cosmological fluid. Determining $w_{0}$ merely provides information about the present-day value of $p / \rho$ but makes no prediction as to what this ratio will do in the future-not even in the near future. (This point is also forcefully made in [12].) For this reason there have been several attempts to observationally determine $w(z)$, the value of $w$ as a function of redshift. See for example [12] and [9-11]. Since $z$ is a function of lookback time $D / c$, this is ultimately equivalent to determining $w(t)=p(t) / \rho(t)$, and implicitly equivalent to reconstructing a phenomenological equation of state $p(\rho)$. I prefer to phrase the discussion directly in terms of the EOS as that will make it clear what parameters have to be physically measured. In terms of the history of the scale factor $a(t)$, it is only when one goes to third order by including the jerk parameter $j_{0}$ that one obtains even a linearized equation of state.

Going one step higher in the expansion, by using the chain rule and the implicit function theorem it is easy to see that

$$
\frac{\mathrm{d}^{2} p}{\mathrm{~d} \rho^{2}}=\frac{\ddot{p}-\kappa \ddot{\rho}}{(\dot{\rho})^{2}} .
$$


More generally $\mathrm{d}^{n} p / \mathrm{d} \rho^{n}$ contains a term linear in $\mathrm{d}^{n} p / \mathrm{d} t^{n}$. Using the Friedmann equations then implies that $\mathrm{d}^{n} p / \mathrm{d} \rho^{n}$ contains a term linear in $\mathrm{d}^{n+2} a / \mathrm{d} t^{n+2}$. Specifically for the first nonlinear term it is relatively straightforward to take explicit time derivatives and so to verify that

$$
\begin{gathered}
\left.\frac{\mathrm{d}^{2} p}{\mathrm{~d} \rho^{2}}\right|_{0}=-\frac{\left(1+k c^{2} /\left[H_{0}^{2} a_{0}^{2}\right]\right)}{6 \rho_{0}\left(1+q_{0}+k c^{2} /\left[H_{0}^{2} a_{0}^{2}\right]\right)^{3}}\left\{s_{0}\left(1+q_{0}\right)+j_{0}\left(1+j_{0}+4 q_{0}+q_{0}^{2}\right)+q_{0}\left(1+2 q_{0}\right)\right. \\
\left.+\left(s_{0}+j_{0}+q_{0}+q_{0} j_{0}\right) \frac{k c^{2}}{H_{0}^{2} a_{0}^{2}}\right\} .
\end{gathered}
$$

In the approximation $H_{0} a_{0} / c \gg 1$ this reduces to

$$
\left.\frac{\mathrm{d}^{2} p}{\mathrm{~d} \rho^{2}}\right|_{0}=-\frac{s_{0}\left(1+q_{0}\right)+j_{0}\left(1+j_{0}+4 q_{0}+q_{0}^{2}\right)+q_{0}\left(1+2 q_{0}\right)}{6 \rho_{0}\left(1+q_{0}\right)^{3}} .
$$

As expected, this second derivative depends linearly on the snap. Higher-order coefficients can certainly be computed but are increasingly complicated and less transparent in their physical interpretation. (Calculations are impractical without the use of some symbolic manipulation package such as Maple.) To now make the connection between the Taylor coefficients of the cosmological EOS and the various parameters appearing in the Hubble law we will need to likewise perform a similar Taylor expansion of the Hubble law.

\section{Hubble law to fourth order in redshift}

Note that this entire section is independent of the use of the Friedmann equations and depends only on the use of a FRW geometry.

The physical distance travelled by a photon that is emitted at time $t_{*}$ and absorbed at the current epoch $t_{0}$ is

$$
D=c \int \mathrm{d} t=c\left(t_{0}-t_{*}\right) .
$$

In terms of this physical distance the Hubble law is exact

$$
1+z=\frac{a\left(t_{0}\right)}{a\left(t_{*}\right)}=\frac{a\left(t_{0}\right)}{a\left(t_{0}-D / c\right)}
$$

but impractical. A more useful result is obtained by performing a fourth-order Taylor series expansion,

$$
\begin{aligned}
\frac{a\left(t_{0}\right)}{a\left(t_{0}-D / c\right)}= & 1+\frac{H_{0} D}{c}+\frac{2+q_{0}}{2} \frac{H_{0}^{2} D^{2}}{c^{2}}+\frac{6\left(1+q_{0}\right)+j_{0}}{6} \frac{H_{0}^{3} D^{3}}{c^{3}} \\
& +\frac{24-s_{0}+8 j_{0}+36 q_{0}+6 q_{0}^{2}}{24} \frac{H_{0}^{4} D^{4}}{c^{4}}+O\left[\left(\frac{H_{0} D}{c}\right)^{5}\right]
\end{aligned}
$$

followed by reversion of the resulting series $z(D) \rightarrow D(z)$ to obtain

$$
\begin{aligned}
D=\frac{c z}{H_{0}}\{1- & {\left[1+\frac{q_{0}}{2}\right] z+\left[1+q_{0}+\frac{q_{0}^{2}}{2}-\frac{j_{0}}{6}\right] z^{2} } \\
- & {\left.\left[1+\frac{3}{2} q_{0}\left(1+q_{0}\right)+\frac{5}{8} q_{0}^{3}-\frac{1}{2} j_{0}-\frac{5}{12} q_{0} j_{0}-\frac{s_{0}}{24}\right] z^{3}+O\left(z^{4}\right)\right\} . }
\end{aligned}
$$

This simple calculation is enough to demonstrate that the jerk shows up at third order in the Hubble law, and the snap at fourth order. Generally, the $O\left(z^{n}\right)$ term in this version of the 
Hubble law will depend on the $n$th time derivative of the scale factor. (Also note that one of the virtues of this version of the Hubble law is that it is completely independent of $k$, the sign of space curvature.)

Unfortunately, physical distance $D$ is typically not the variable in terms of which the Hubble law is observationally presented. That role is more typically played by the 'luminosity distance', $d_{L}$. For instance, Weinberg defines [1]

$$
(\text { energy flux })=\frac{L}{4 \pi d_{L}^{2}} .
$$

Let the photon be emitted at $r$-coordinate $r=0$ at time $t_{*}$, and absorbed at $r$-coordinate $r=r_{0}$ at time $t_{0}$. Then it is a purely geometrical result that

$$
d_{L}=a\left(t_{0}\right)^{2} \frac{r_{0}}{a\left(t_{*}\right)}=\frac{a_{0}}{a\left(t_{0}-D / c\right)}\left(a_{0} r_{0}\right) .
$$

Thus to calculate $d_{L}(D)$ we need $r_{0}(D)$. Recall that for a null geodesic in a FRW universe

$$
\int_{t_{*}}^{t_{0}} \frac{c \mathrm{~d} t}{a(t)}=\int_{0}^{r_{0}} \frac{\mathrm{d} r}{\sqrt{1-k r^{2}}}=f\left(r_{0}\right) .
$$

But

$$
f\left(r_{0}\right)= \begin{cases}\sin ^{-1} r_{0} & k=+1 \\ r_{0} & k=0 \\ \sinh ^{-1} r_{0} & k=-1\end{cases}
$$

therefore

$$
r_{0}(D)=f^{-1}\left(\int_{t_{*}(D)}^{t_{0}} \frac{c \mathrm{~d} t}{a(t)}\right)=f^{-1}\left(\int_{t_{0}-D / c}^{t_{0}} \frac{c \mathrm{~d} t}{a(t)}\right) .
$$

To be explicit

$$
r_{0}(D)= \begin{cases}\sin \left(\int_{t_{0}-D / c}^{t_{0}} \frac{c \mathrm{~d} t}{a(t)}\right) & k=+1, \\ \int_{t_{0}-D / c}^{t_{0}} \frac{c \mathrm{~d} t}{a(t)} & k=0, \\ \sinh \left(\int_{t_{0}-D / c}^{t_{0}} \frac{c \mathrm{~d} t}{a(t)}\right) & k=-1 .\end{cases}
$$

We can now Taylor series expand for 'short' distances. First note that

See endnote 2

$r_{0}(D)=\left[\int_{t_{0}-D / c}^{t_{0}} \frac{c \mathrm{~d} t}{a(t)}\right]-\frac{k}{3 !}\left[\int_{t_{0}-D / c}^{t_{0}} \frac{c \mathrm{~d} t}{a(t)}\right]^{3}+O\left(\left[\int_{t_{0}-D / c}^{t_{0}} \frac{c \mathrm{~d} t}{a(t)}\right]^{5}\right)$,

and now expand the integral to third order. (We can check, a posteriori, that this retains sufficient accuracy in the $d_{L} \leftrightarrow D$ conversion for determining the Hubble law to fourth order.) Then

$$
\begin{aligned}
\int_{t_{0}-D / c}^{t_{0}} \frac{c \mathrm{~d} t}{a(t)}= & \int_{t_{0}-D / c}^{t_{0}} \frac{c \mathrm{~d} t}{a_{0}}\left\{1+H_{0}\left(t_{0}-t\right)+\left[\frac{2+q_{0}}{2} H_{0}^{2}\right]\left(t_{0}-t\right)^{2}\right. \\
& \left.+\left[\frac{6\left(1+q_{0}\right)+j_{0}}{6} H_{0}^{3}\right]\left(t_{0}-t\right)^{3}+O\left[\left(t_{0}-t\right)^{4}\right]\right\}
\end{aligned}
$$




$$
\begin{aligned}
= & \frac{c}{a_{0}}\left\{D / c+\frac{1}{2} H_{0}(D / c)^{2}+\left[\frac{2+q_{0}}{6} H_{0}^{2}\right](D / c)^{3}\right. \\
& \left.+\left[\frac{6\left(1+q_{0}\right)+j_{0}}{24} H_{0}^{3}\right](D / c)^{4}+O\left[H_{0}^{4}(D / c)^{5}\right]\right\} \\
= & \frac{D}{a_{0}}\left\{1+\frac{1}{2} \frac{H_{0} D}{c}+\left[\frac{2+q_{0}}{6}\right]\left(\frac{H_{0} D}{c}\right)^{2}+\left[\frac{6\left(1+q_{0}\right)+j_{0}}{24}\right]\left(\frac{H_{0} D}{c}\right)^{3}\right. \\
& \left.+O\left[\left(\frac{H_{0} D}{c}\right)^{4}\right]\right\} .
\end{aligned}
$$

The conversion from physical distance travelled to $r$ coordinate traversed is given by

$$
\begin{aligned}
r_{0}(D)=\frac{D}{a_{0}}\{1 & +\frac{1}{2} \frac{H_{0} D}{c}+\frac{1}{6}\left[2+q_{0}-\frac{k c^{2}}{H_{0}^{2} a_{0}^{2}}\right]\left(\frac{H_{0} D}{c}\right)^{2} \\
& \left.+\frac{1}{24}\left[6\left(1+q_{0}\right)+j_{0}-6 \frac{k c^{2}}{H_{0}^{2} a_{0}^{2}}\right]\left(\frac{H_{0} D}{c}\right)^{3}+O\left[\left(\frac{H_{0} D}{c}\right)^{4}\right]\right\} .
\end{aligned}
$$

Combining these formulae we find the luminosity distance as a function of $D$, the physical distance travelled is

$$
\begin{aligned}
d_{L}(D)=D\{1 & +\frac{3}{2}\left(\frac{H_{0} D}{c}\right)+\frac{1}{6}\left[11+4 q_{0}-\frac{k c^{2}}{H_{0}^{2} a_{0}^{2}}\right]\left(\frac{H_{0} D}{c}\right)^{2} \\
& \left.+\frac{1}{24}\left[50+40 q_{0}+5 j_{0}-10 \frac{k c^{2}}{H_{0}^{2} a_{0}^{2}}\right]\left(\frac{H_{0} D}{c}\right)^{3}+O\left[\left(\frac{H_{0} D}{c}\right)^{4}\right]\right\} .
\end{aligned}
$$

Now using the series expansion for $D(z)$ we finally derive the luminosity-distance version of the Hubble law:

$$
\begin{aligned}
d_{L}(z)=\frac{c z}{H_{0}}\{1 & +\frac{1}{2}\left[1-q_{0}\right] z-\frac{1}{6}\left[1-q_{0}-3 q_{0}^{2}+j_{0}+\frac{k c^{2}}{H_{0}^{2} a_{0}^{2}}\right] z^{2}+\frac{1}{24}\left[2-2 q_{0}\right. \\
& \left.\left.-15 q_{0}^{2}-15 q_{0}^{3}+5 j_{0}+10 q_{0} j_{0}+s_{0}+\frac{2 k c^{2}\left(1+3 q_{0}\right)}{H_{0}^{2} a_{0}^{2}}\right] z^{3}+O\left(z^{4}\right)\right\} .
\end{aligned}
$$

The first two terms given above are Weinberg's version of the Hubble law (his equation (14.6.8)). The third term is equivalent to that obtained by Chiba and Nakamura [8]. The fourth-order term appears to be new, and (as expected) depends linearly on the snap. From the derivation above it is now clear that the $O\left(z^{n}\right)$ term in this luminosity-distance version of the Hubble law will also depend on the $n$th time derivative of the scale factor.

It is important to realize that this Hubble law, and indeed the entire discussion of this section, is completely model-independent-it assumes only that the geometry of the universe is well approximated by a FRW cosmology but does not invoke the Einstein field equations (Friedmann equation) or any particular matter model. Note that in comparison to the $D(z)$ Hubble law, this $d_{L}(z)$ Hubble law first differs in the coefficient of the $O\left(z^{2}\right)$ term-you will still get the same Hubble parameter, but if you are not sure which definition of 'distance' you are using you may mis-estimate the higher-order coefficients (deceleration, jerk and snap). The jerk $j_{0}$ first shows up in the Hubble law at third order $\left(\right.$ order $z^{3}$ ); but this was one of the parameters we needed to make the lowest-order estimate for the slope of the EOS.

Warning. Not all authors use the same definition of the luminosity distance. In particular D'Inverno uses a definition that differs from Weinberg's by an extra factor of $(1+z)^{2}[18]$. 
Weinberg's definition as presented above appears to be the most standard, but if necessary the conversion is straightforward.

\section{A specific 'a priori' model: incoherent mixture of $w$-matter}

Though the philosophy so far has been to avoid committing ourselves to any particular matter model, it is useful for comparison purposes to see how these ideas impact on the most popular models. A particularly common a priori model for the cosmological fluid is an incoherent mixture of various forms of $w$-matter with each component satisfying a zero-offset equation of state:

$$
p_{i}=w_{i} \rho_{i}
$$

Integrating the conservation equation independently for each component of the mixture yields $p_{i}=p_{0 i}\left(a / a_{0}\right)^{-3\left(1+w_{i}\right)}=\rho_{0 i} w_{i}\left(a / a_{0}\right)^{-3\left(1+w_{i}\right)}=\rho_{c} \Omega_{0 i} w_{i}\left(a / a_{0}\right)^{-3\left(1+w_{i}\right)}$.

This model is sufficiently general to contain dust, radiation, cosmological constant and standard forms of quintessence. Then

$$
w_{0}=\frac{\sum_{i} \Omega_{0 i} w_{i}}{\sum_{i} \Omega_{0 i}}=\bar{w}
$$

is simply the weighted average value of $w$. (A sum of $w_{i}$ over all matter components $i$, weighted by their present-day contribution to the $\Omega$ parameter.) Similarly

$$
\kappa_{0}=\frac{\mathrm{d} p /\left.\mathrm{d} a\right|_{0}}{\mathrm{~d} \rho /\left.\mathrm{d} a\right|_{0}}=\frac{\sum_{i} \Omega_{0 i} w_{i}\left(1+w_{i}\right)}{\sum_{i} \Omega_{0 i}\left(1+w_{i}\right)}=\frac{\overline{w^{2}}+\bar{w}}{1+\bar{w}}=\frac{\sigma_{w}^{2}}{1+\bar{w}}+\bar{w} .
$$

That is, $w_{0}$ (and hence $q_{0}$ ) provides information about the weighted average value of the $w_{i}$, while $\kappa_{0}$ (and hence $j_{0}$ ) provides information about how much spread there is in the various $w_{i}$. Uncertainties in the jerk parameter $j_{0}$ (which in a generic model manifest themselves as uncertainties in the slope parameter $\kappa_{0}$ ) in this specific model show up as difficulty in determining the weighting parameters $\Omega_{0 i}$. (For similar comments, see [12].) Note that the positivity of $\sigma_{w}^{2}$ implies a constraint

$$
(1+\bar{w})\left(\kappa_{0}-\bar{w}\right)>0 .
$$

If this inequality observationally fails, it means that the cosmological fluid cannot be described by any possible linear combination of $w$-matter. In the approximation $H_{0} a_{0} / c \gg 1$ this reduces to

$$
j_{0}>q_{0}\left(1-q_{0}\right) .
$$

At the next highest level in the Taylor expansion, again making use of the implicit function theorem, we have

$$
\frac{\mathrm{d}^{2} p}{\mathrm{~d} \rho^{2}}=\frac{p^{\prime \prime}-\kappa \rho^{\prime \prime}}{\left(\rho^{\prime}\right)^{2}}
$$

After a brief computation this leads to

$$
\left.\rho_{0} \frac{\mathrm{d}^{2} p}{\mathrm{~d} \rho^{2}}\right|_{0}=\frac{\overline{w^{3}}(1+\bar{w})+\overline{w^{2}}\left(1-\bar{w}-\overline{w^{2}}\right)-\bar{w}^{2}}{(1+\bar{w})^{3}} .
$$

In terms of standard deviation and skewness,

$$
\mathcal{S}_{w}=\overline{(w-\bar{w})^{3}}=\overline{w^{3}}-3 \overline{w^{2}} \bar{w}+2 \bar{w}^{3}=\overline{w^{3}}-3 \sigma_{w}^{2}-\bar{w}^{3},
$$


this yields

$$
\left.\rho_{0} \frac{\mathrm{d}^{2} p}{\mathrm{~d} \rho^{2}}\right|_{0}=\frac{\mathcal{S}_{w}(1+\bar{w})-\sigma_{w}^{4}-2 \sigma_{w}^{2} \bar{w}^{2}-2 \sigma_{w}^{2}-4 \sigma^{2} \bar{w}-2 \bar{w}^{3}-2 \bar{w}^{4}}{(1+\bar{w})^{3}} .
$$

Thus $\mathrm{d}^{2} p /\left.\mathrm{d} \rho^{2}\right|_{0}$ is related to skewness in the distribution of the $w_{i}$. The general message to be extracted here is that the $n$th Taylor coefficient in the EOS depends linearly on the $(n+1)$ th $\Omega$-weighted moment of the $w_{i}$.

\section{The observational situation}

As of March 2004, the observational situation is best summarized by the dataset described in the recent paper by Riess et al [19]. (See also the brief analysis in Caldwell and Kamionkowski [20]). The Riess et al analysis presupposes a $k=0$ spatially flat universe (effectively, they adopt the inflationary paradigm and use the approximation $\left.c /\left(H_{0} a_{0}\right) \ll 1\right)$. They report that the jerk $j_{0}$ is positive at the $92 \%$ confidence level based on their 'gold' dataset, and is positive at the $95 \%$ confidence level based on their 'gold+silver' dataset. No explicit upper bounds are given for the jerk, nor are any constraints placed on the snap $s_{0}$. The allowed region (for their preferred parametrization of the data in terms of $\left.[\mathrm{d} q / \mathrm{d} z]_{0}\right)$ is presented in their figure 5 , and can usefully be summarized as follows: the allowed region is a narrow ellipse approximately centred along the line

$$
\frac{\mathrm{d} q}{\mathrm{~d} z}=-1-\frac{7}{2} q_{0}
$$

and bounded by the boxes

$q_{0} \in(-1.3,-0.2),\left.\quad \frac{\mathrm{d} q}{\mathrm{~d} z}\right|_{0} \in(-0.2,+3.8) \quad(99 \%$ confidence, 'gold'),

and

$q_{0} \in(-1.4,-0.3),\left.\quad \frac{\mathrm{d} q}{\mathrm{~d} z}\right|_{0} \in(+0.0,+3.9) \quad(99 \%$ confidence, 'gold + silver').

To extract more detailed information, one needs to translate from $[\mathrm{d} q / \mathrm{d} z]_{0}$ to jerk $j_{0}$. Working to third order in redshift it is straightforward to calculate $(k=0)$ :

$$
d_{L}(z)=\frac{c z}{H_{0}}\left\{1+\frac{1-q_{0}}{2} z-\frac{1-q_{0}^{2}+[\mathrm{d} q / \mathrm{d} z]_{0}}{6} z^{2}+O\left(z^{3}\right)\right\},
$$

and so obtain

$$
j_{0}=q_{0}+2 q_{0}^{2}+\left.\frac{\mathrm{d} q}{\mathrm{~d} z}\right|_{0}
$$

Using $w_{0}=-\left(1-2 q_{0}\right) / 3$ it is then easy to determine

$w_{0} \in(-1.2,-0.5), \quad j_{0} \in(-0.3,+5.9) \quad$ (99\% confidence, 'gold'),

and

$w_{0} \in(-1.3,-0.5), \quad j_{0} \in(-0.1,+6.4) \quad$ (99\% confidence, 'gold + silver').

Note that even at the $68 \%$ level both datasets exhibit a thin sliver of parameter space compatible with $q_{0}<-1$. (That is, $w_{0}<-1$, so that the grand total of all stress-energy contributing to the cosmological fluid is 'phantom'.) Also note that the upper bounds on the jerk are comparatively weak. 
Now consider the linearized equation of state using

$$
\kappa_{0}=-\frac{1}{3} \frac{1-j_{0}}{1+q_{0}}
$$

This parameter is very poorly constrained

$$
\kappa_{0} \in(-\infty,-5.5) \cup(-0.5,+\infty) \quad \text { (99\% confidence, 'gold'), }
$$

and

$\kappa_{0} \in(-\infty,-4.5) \cup(-0.5,+\infty) \quad$ (99\% confidence, 'gold+silver').

Note that very little of the real line is excluded, and that the entire positive axis is allowed by the data. Since this might at first be a bit surprising, let me explain the basic reason for this behaviour: if the permissible region contains a subset compatible with $j_{0} \neq 1$ and $q_{0}=-1$, then this implies that a subset of the permissible region is compatible with $\kappa_{0}= \pm \infty$. That is, whenever there is a part of parameter space compatible with phantom matter, then $\kappa_{0} \rightarrow \pm \infty$ at the edge of the phantom region.

Similarly, as long as the permissible region contains a subset compatible with $j_{0}=1$ and $q_{0} \neq-1$, this implies that a subset of the permissible region is compatible with $\kappa_{0}=0$. Now $j_{0}=1$ is equivalent to

$$
\left.\frac{\mathrm{d} q}{\mathrm{~d} z}\right|_{0}=\left(1+q_{0}\right)\left(1-2 q_{0}\right)
$$

and it is easy to see that a subset of the Riess et al permissible region is compatible with this constraint. With both these conditions being satisfied the entire positive real line is allowed for $\kappa_{0}$. The portion of the negative real line attached to $-\infty$ is associated with phantom matter, while the portion of the negative real line attached to the origin is associated with non-phantom matter $\left(w_{0} \geqslant-1\right)$ with the peculiar property $\mathrm{d} p / \mathrm{d} \rho<0$.

It is also easy to see why at least some values of $\kappa_{0}$ are excluded. If the permissible region contains the point $q_{0}=-1, j_{0}=+1$ (equivalently $q_{0}=-1,[\mathrm{~d} q / \mathrm{d} z]_{0}=0$ ) then, since this corresponds to $\kappa_{0}$ taking on the indeterminate value $0 / 0$, it is easy to convince oneself that for any open set surrounding this point the computed values of $\kappa_{0}$ completely cover the entire real line-both positive and negative. But the point $q_{0}=-1, j_{0}=+1\left(q_{0}=-1,[\mathrm{~d} q / \mathrm{d} z]_{0}=0\right)$ is in fact excluded from both gold and gold+silver datasets at more than $99 \%$ confidence, which is ultimately the reason that at least some values of $\kappa_{0}$ can be excluded. However, it should be emphasized that the constraints on $\kappa_{0}$ are best described as extremely weak.

A slightly different analysis (using the same raw data analysed in somewhat different fashion) is presented by Caldwell and Kamionkowski [20]. Their result can usefully be summarized (again assuming $k=0$ ) as

$$
q_{0} \in(-1.1,-0.2), \quad j_{0} \in(-0.5,+3.9) \quad(95 \% \text { confidence }),
$$

whence

$$
w_{0} \in(-1.1,-0.5) \quad(95 \% \text { confidence })
$$

and

$$
\kappa_{0} \in(-\infty,-10) \cup(-0.6,+\infty) \quad \text { (95\% confidence) } .
$$

While the numbers are slightly different, the overall message is the same: the jerk is relatively weakly constrained and $\kappa_{0}$ is very poorly constrained.

If one wishes to constrain $\kappa_{0}$ to at least lie in some bounded region of the real line, then one would need to seek improved data that might exclude the possibility $q_{0}=-1\left(w_{0}=-1\right)$. (This would then be the death-knell for phantom matter.) Similarly, if one wishes to constrain 
$\kappa_{0}$ to at least lie in the 'physically most reasonable' region (some bounded region of the positive real line), one would need to seek improved data that might exclude the possibility $j_{0}<1$. Obtaining a dataset of such quality would be extremely challenging: assuming no change in the location of the centre of the currently determined permissible region, this would correspond to contracting the $99 \%$ confidence intervals inwards to lie somewhere inside the current location of the $68 \%$ confidence intervals. This corresponds to approximately a three-fold decrease in the standard deviations obtained when fitting the Hubble law to the dataset; despite the advances in 'precision cosmology', this appears well beyond current (or even reasonably foreseeable) capabilities.

Furthermore, one should note that the above considerations are subject to having additional data (or theoretical prejudices) available to justify setting $k=0$ (or more precisely, $\left.c /\left(H_{0} a_{0}\right) \ll 1\right)$. In the absence of such data, the third-order term in the luminosity-distance Hubble law only implies [20]

$q_{0} \in(-1.1,-0.2), \quad j_{0}+\frac{k c^{2}}{H_{0}^{2} a_{0}^{2}} \in(-0.5,+3.9) \quad(95 \%$ confidence $)$.

Constraints on the equation of state, $\kappa_{0}$, are now even weaker.

Note that appealing to the quartic term in the luminosity-distance version of the Hubble law will not help, as that fourth-order term brings in an additional free parameter (the snap $s_{0}$ ), so that there are still more free parameters than there are coefficients that can be measured. To sidestep this particular problem the two possibilities are: (1) the traditional one, which is to find additional data, above and beyond the Hubble law, that somehow constrain the space curvature $k / a_{0}^{2}$. (2) More challengingly, using a modified Hubble law that is independent of space curvature $k / a_{0}^{2}$. For instance, the 'physical distance travelled' version of the Hubble law $D(z)$ presented in equation (33) is completely independent of space curvature - the challenge in this approach is to find a useful way of observationally measuring $D$.

\section{Discussion}

There are currently many different models for the cosmological fluid under active consideration. Though these models often make dramatically differing predictions in the distant past (e.g., a 'bounce') or future (e.g., a 'big rip') there is considerable degeneracy among the models in that many physically quite different models are compatible with present day observations. To understand the origin of this degeneracy I have chosen to rephrase the question in terms of a phenomenological approach where cosmological observations are used to construct an 'observed' equation of state. The key result is that even at the linearized level, determining the slope of the EOS requires information coming from the third-order term in the Hubble law. Unfortunately, while the experimental determinations of the parameters appearing in the Hubble law are certainly improving, we are still somewhat limited in what we can say concerning the third-order term. Despite the fact that some parameters in cosmology are now known to high accuracy, other parameters can still only be crudely bounded [21]. In particular, the jerk is relatively poorly bounded, and as a consequence direct observational constraints on the cosmological EOS (in the form of measurements of $\kappa_{0}=[\mathrm{d} p / \mathrm{d} \rho]_{0}$ ) are currently extremely poor and are likely to remain poor for the foreseeable future.

\section{Acknowledgments}

This Research was supported by the Marsden Fund administered by the Royal Society of New Zealand. I wish to thank T Chiba, T Padmanabhan, A Riess and V Sahni for their comments. 


\section{References}

[1] Weinberg S 1972 Gravitation and Cosmology: Principles and Applications of the General Theory of Relativity (New York: Wiley)

[2] Misner C W, Thorne K S and Wheeler J A 1973 Gravitation (San Francisco: Freeman)

[3] Peebles P J E 1993 Principles of Physical Cosmology (Princeton)

[4] Morris M S and Thorne K S 1988 Wormholes in space-time and their use for interstellar travel: a tool for teaching general relativity Am. J. Phys. 56395

[5] Molina-París C and Visser M 1999 Minimal conditions for the creation of a Friedman-Robertson-Walker universe from a 'bounce' Phys. Lett. B 45590 (Preprint gr-qc/9810023)

[6] Hochberg D, Molina-París C and Visser M 1999 Tolman wormholes violate the strong energy condition Phys. Rev. D 59044011 (Preprint gr-qc/9810029)

[7] Caldwell R R, Kamionkowski M and Weinberg N N 2003 Phantom energy and cosmic doomsday Phys. Rev. Lett. 91071301 (Preprint astro-ph/0302506)

Kallosh R, Kratochvil J, Linde A, Linder E V and Shmakova M 2003 Observational bounds on cosmic doomsday Preprint astro-ph/0307185

[8] Chiba T and Nakamura T 1998 The luminosity distance, the equation of state, and the geometry of the universe Prog. Theor. Phys. 1001077 (Preprint astro-ph/9808022)

[9] Sahni V, Saini T D, Starobinsky A A and Alam U 2003 Statefinder-a new geometrical diagnostic of dark energy Pisma Zh. Eksp. Teor. Fiz. 77249 (Preprint astro-ph/0201498)

Sahni V, Saini T D, Starobinsky A A and Alam U 2003 JETP Lett. 77201 (Engl. Transl.)

[10] Sahni V 2002 Exploring dark energy using the statefinder Preprint astro-ph/0211084

[11] Alam U, Sahni V, Saini T D and Starobinsky A A 2003 Exploring the expanding universe and dark energy using the statefinder diagnostic Preprint astro-ph/0303009

[12] Padmanabhan T and Choudhury T R 2003 A theoretician's analysis of the supernova data and the limitations in determining the nature of dark energy Mon. Not. Roy. Astron. Soc. 344823 (Preprint astro-ph/0212573)

[13] Padmanabhan T 2003 Cosmological constant: the weight of the vacuum Phys. Rep. 380235 (Preprint hepth/0212290)

[14] Cramer J G, Forward R L, Morris M S, Visser M, Benford G and Landis G A 1995 Natural wormholes as gravitational lenses Phys. Rev. D 513117 (Preprint astro-ph/9409051)

[15] Visser M 1995 Lorentzian Wormholes: From Einstein To Hawking (Reading, MA: AIP Press)

[16] Barceló C and Visser M 2002 Twilight for the energy conditions? Int. J. Mod. Phys. D 111553 (Preprint gr-qc/0205066)

[17] Visser M 2002 The quantum physics of chronology protection Preprint gr-qc/0204022

[18] D’Inverno R 1992 Introducing Einstein's Relativity (Oxford)

[19] Riess A G et al 2004 Type Ia supernova discoveries at $z>1$ from the Hubble space telescope: evidence for past deceleration and constraints on dark energy evolution Preprint astro-ph/0402512

[20] Caldwell R R and Kamionkowski M 2004 Expansion, geometry, and gravity Preprint astro-ph/0403003

[21] Bridle S L, Lahav O, Ostriker J P and Steinhardt P J 2003 Precision cosmology? not just yet Science 2991532 (Preprint astro-ph/0303180) 


\section{Endnotes}

(1) Author: Please provide PACS numbers in full form.

(2) Author: The meaning of 'Taylor series expand' is unclear. Please check.

(3) Author: Please provide publisher in references [3] and [18]. 